\title{
4G model of fractional charge strong-weak super symmetry
}

\author{
Seshavatharam U.V.S ${ }^{1,2}$ and Lakshminarayana $S^{3}$ \\ ${ }^{1}$ Honorary faculty, I-SERVE, Survey no-42, Hitech city, Hyderabad-84,Telangana, INDIA \\ ${ }^{2}$ Asst. Mgr., QA Dept, DIP-SPUN, Srikalahasthi Pipes Ltd, Srikalahasthi-517641, AP, INDIA \\ ${ }^{3}$ Dept. of Nuclear Physics, Andhra University, Visakhapatnam-03, AP, INDIA \\ Emails: seshavatharam.uvs@gmail.com (and) 1nsrirama@gmail.com \\ Orcid numbers : 0000-0002-1695-6037 (and) 0000-0002-8923-772X
}

\begin{abstract}
To understand the mystery of final unification, in our earlier publications, we proposed that, 1) There exist three atomic gravitational constants associated with electroweak, strong and electromagnetic interactions; and 2) There exists a strong interaction elementary charge $\left(e_{s}\right)$ in such a way that, it's squared ratio with normal elementary charge is close to inverse of the strong coupling constant. In this context, starting from lepton rest masses to stellar masses, we have developed many interesting and workable relations. We noticed that, electroweak field seems to be operated by a primordial massive fermion of rest energy $585 \mathrm{GeV}$. It can be considered as the zygote of all elementary particles and galactic dark matter. Proceeding further, with a characteristic fermion-boson mass ratio of 2.27, quarks can be classified into quark fermions and quark bosons. Considering strong charge conservation and electromagnetic charge conservation, fractional charge quark fermions and quark bosons can be understood. Quark fermions that generate observable massive baryons can be called as Fluons. Quark bosons that generate observable mesons can be called as Bluons. By considering a new hadronic fermion of rest energy $103.4 \mathrm{GeV}$, rest masses of fluons and bluons can be estimated and there by baryon masses and meson masses can be estimated.
\end{abstract}

Key words: Four gravitational constants; Super symmetry; strong charge; strong coupling constant; quark fermions, quark bosons, fluons; bluons; baryons; mesons;

\begin{tabular}{|c|c|c|c|}
\hline \multicolumn{4}{|c|}{ Nomenclature } \\
\hline 1) & Newtonian gravitational constant $=G_{N}$ & 24) & Mean stable mass number $=A_{m}$ \\
\hline 2) & Electromagnetic gravitational constant $=G_{e}$ & 25) & Nuclear binding energy $=B_{(A, Z)}$ \\
\hline 3) & Nuclear gravitational constant $=G_{s}$ & & Nuclear binding energy coefficient $=B_{0}$ \\
\hline 4) & Weak gravitational constant $=G_{W}$ & 27) & Coefficients connected with nuclear stability \\
\hline 5) & Fermi's weak coupling constant $=G_{F}$ & & and binding energy $=\left(k_{1}, k_{2}\right)$ \\
\hline 6) & New electroweak fermion $=M_{w f}$ & 28) & Mass limit of stellar object $=M_{X}$ \\
\hline 7) & New electroweak boson $=M_{w b}$ & 29) & Characteristic ratio associated with charged \\
\hline 8) & Reduced Planck's constant $=\hbar$ & & $\sqrt{4 \pi \varepsilon_{0} G_{e} m_{e}^{2}}$ \\
\hline $\begin{array}{l}\text { 9) } \\
\text { 10) }\end{array}$ & $\begin{array}{l}\text { Speed of light }=c \\
\text { Strong counling constant }=\alpha\end{array}$ & & leptons $=\sqrt{\frac{e}{e^{2}} \cong \gamma}$ \\
\hline 11) & Elementary charge $=e$ & $30)$ & Mass of charged baby lepton $=\left(m_{1}\right)^{ \pm}$ \\
\hline 12) & Strong elementary charge $=e$ & & (1) \\
\hline 13) & & 31) & Neutron nire time $=l_{n}$ \\
\hline 13) & Mass of proton $=m_{p}$ & 32) & Mass of basic quark $=m_{q}$ \\
\hline 14) & Mass of neutron $=m_{n}$ & 33) & SUSY Fermion-boson mass ratio $=\Psi$ \\
\hline 15) & Mass of electron $=m_{e}$ & 34) & Mass of quark fermion $=m_{q f}$ \\
\hline 16) & Mass of Up quark $=m_{u}$ & $35)$ & Mass of quark boson $=m_{q b}$ \\
\hline 17) & Mass of Down quark $=m_{d}$ & 36) & Baryon mass generator $=M_{h f}$ \\
\hline 18) & Bohr Radius $=a_{0}$ & 37) & Meson mass generator $=M_{h b}$ \\
\hline 19) & Schwarzschild radius of $m_{p}=R_{p}$ & 38) & Mass of Fluon $=M_{q f}$ \\
\hline 20) & Nuclear charge radius $=R_{(Z, A)}$ & 39) & Mass of Bluon $=M_{q b}$ \\
\hline 21) & Schwarzschild radius of $M_{w}=R_{w}$ & 40) & Mass of neutral electroweak boson $=M_{z}$ \\
\hline 22) & Schwarzschild radius of $m_{e}=R_{e}$ & 41) & Mass of charged electroweak boson $=M_{W}$ \\
\hline & Schwarzschild radius of atom $=R_{\text {atom }}$ & 42) & Mass of neutral Higg's boson $=M_{H}$ \\
\hline
\end{tabular}




\section{Introduction}

Even though celestial objects that show gravity are confirmed to be made up of so many atoms, so far scientists could not find any relation in between gravity and the atomic interactions at quantum gravity level $[1,2]$. Black hole temperature point of view [3], strong interaction point of view [4-7] and electroweak interaction point of view [8], scientists found very interesting similarities in between gravity and quantum phenomena. Quantum cosmology point of view [9] and nuclear quantum gravity point of view [10-20], authors could develop workable ideas, concepts and relations. On a whole, workability is still lagging. It clearly indicates that, there is something wrong in our notion of understanding or there is something missing in developing the unified physical concepts and needs a critical review at fundamental level. In this context, we hope that, electroweak scale $[21,22,23]$ can certainly yield useful stuff.

\section{Motivating concepts}

To develop new and workable ideas, we wish to highlight the following points.

1) During cosmic evolution, if one is willing to give equal importance to Higgs boson and Planck mass in understanding the massive origin of elementary particles and observed matter [24,25], then it seems quite logical to expect a common relation in between Planck scale and Electroweak scale.

2) Whether particle's massive nature is due to electromagnetism or gravity or weak interaction or strong interaction or cosmic dust or dark matter [26] or something else, is unclear.

3) Without understanding the massive nature, it is not reasonable to classify the field created by any elementary particle.

4) All the four interactions seem to be associated with $(\hbar)$.

5) Nobody knows the mystery of $(\hbar)$ which seems to be a basic measure of angular momentum [27,28,29,30].

6) Nobody knows the mystery of existence, stability and behavior of 'proton' or 'electron'.

7) 'Mass' is a basic property of space-time curvature and basic ingredient of angular momentum.
8) Atoms are mainly characterized by protons and electrons.

9) 'Free neutron' is an unstable particle.

\section{Basic assumptions}

1) There exists a characteristic electroweak fermion of rest energy [18], $M_{w f} c^{2} \cong 584.725 \mathrm{GeV}$.

2) $M_{w f}$ can be considered as the zygote of all elementary particles.

3) Fermi's weak coupling constant $\left(G_{F}\right)[29,30,31]$ can be considered as the basic unified coupling constant.

4) There exists a strong interaction elementary charge $\left(e_{s}\right)$ in such a way that, it's squared ratio with normal elementary charge is close to inverse of the strong coupling constant.

5) Each atomic interaction is associated with a characteristic gravitational coupling constant.

$$
\begin{aligned}
& G_{e} \cong 2.374335 \times 10^{37} \mathrm{~m}^{3} \mathrm{~kg}^{-1} \mathrm{sec}^{-2} \\
& G_{S} \cong 3.329561 \times 10^{28} \mathrm{~m}^{3} \mathrm{~kg}^{-1} \mathrm{sec}^{-2} \\
& G_{w} \cong 2.909745 \times 10^{22} \mathrm{~m}^{3} \mathrm{~kg}^{-1} \mathrm{sec}^{-2} \\
& G_{N} \cong 6.679855 \times 10^{-11} \mathrm{~m}^{3} \mathrm{~kg}^{-1} \mathrm{sec}^{-2}
\end{aligned}
$$

\section{Characteristic unified relations}

Based on the above points, we propose the following new and workable relations.

$$
\begin{aligned}
& \hbar c \cong G_{w} M_{w f}^{2} \cong \sqrt{G_{F}\left(\frac{c^{4}}{4 G_{w}}\right)} \\
& \Rightarrow \hbar \cong \frac{G_{w} M_{w f}^{2}}{c} \cong \sqrt{\frac{G_{F} c^{2}}{4 G_{w}}}
\end{aligned}
$$

where $\left(\frac{c^{4}}{4 G_{w}}\right) \cong 6.9401 \times 10^{10} \mathrm{~N}$ is the characteristic force associated with electroweak interaction.

$$
\begin{gathered}
m_{e} \cong\left(\frac{G_{w}}{G_{s}}\right) M_{w f} \\
m_{p} \cong\left(\frac{G_{s}}{G_{w}}\right)\left(\frac{G_{s}}{G_{e}}\right) M_{w} \cong\left(\frac{G_{s}^{2}}{G_{w} G_{e}}\right) M_{w f}
\end{gathered}
$$




$$
\frac{m_{p}}{m_{e}} \cong \frac{G_{s}^{3}}{G_{w}^{2} G_{e}}
$$

5. Specific unified relations connected with $\left(G_{e}, G_{s}, G_{w}, G_{N}\right)$

With reference to Newtonian gravitational constant [32-39],

$$
\begin{array}{r}
\left(\frac{m_{p}}{m_{e}}\right)^{10} \cong\left(\frac{G_{w}}{G_{N}}\right) \\
\exp \left(\frac{1}{\alpha_{s}^{2}}\right) \cong\left(\frac{G_{w}}{G_{N}}\right)
\end{array}
$$

where $\alpha_{\mathrm{s}}=$ Strong coupling constant [29,31]

$$
\begin{gathered}
\frac{m_{p}}{m_{e}} \cong\left(\frac{G_{s}}{G_{e}^{1 / 3} G_{N}^{2 / 3}}\right)^{1 / 7} \\
\frac{M_{w f}}{m_{e}} \cong \frac{G_{w}^{5 / 2} G_{e}^{5 / 3}}{G_{s}^{4} G_{N}^{1 / 6}} \\
\frac{M_{w f}}{m_{p}} \cong \frac{G_{s}^{1 / 2} G_{e}^{1 / 6} G_{N}^{1 / 12}}{G_{w}^{3 / 4}} \\
\frac{m_{p}}{m_{e}} \cong \frac{G_{w}^{13 / 4} G_{e}^{3 / 2}}{G_{s}^{9 / 2} G_{N}^{1 / 4}}
\end{gathered}
$$

Based on these relations,

$$
G_{N} \cong\left(\frac{m_{e}}{m_{p}}\right)^{4} \frac{G_{w}^{13} G_{e}^{6}}{G_{s}^{18}}
$$

Based on the nuclear experiments and astrophysical observations,

1) $\left(G_{w}\right)$ can be estimated from relation (1).

2) $\left(G_{s}\right)$ can be estimated from relations (12-14).

3) $\left(G_{e}\right)$ can be estimated from relation (30).

\section{Specific unified relations connected with nuclear radius and Bohr radius}

Characteristic Schwarzschild radius of proton and Schwarzschild radius of atom can be addressed with the following relations.
$R_{p} \cong \frac{2 G_{s} m_{p}}{c^{2}} \cong 1.2393 \mathrm{fm}$

$=$ Characteristic nuclear charge radius $[40,41]$

$$
\begin{aligned}
R_{(Z, A)} & \cong\left\{Z^{1 / 3}+(\sqrt{Z(A-Z)})^{1 / 3}\right\}\left(\frac{G_{s} m_{p}}{c^{2}}\right) \\
& =\text { Nuclear charge radius [42] }
\end{aligned}
$$

$$
\begin{aligned}
a_{0} & \cong\left(\frac{4 \pi \varepsilon_{0} G_{e} m_{e}^{2}}{e^{2}}\right)\left(\frac{G_{s} m_{p}}{c^{2}}\right) \cong 5.2918 \times 10^{-11} \mathrm{~m} \\
& =\text { Bohr radius of Hydrogen atom }[28]
\end{aligned}
$$

\section{Specific unified relations connected with proton-electron mass ratio}

With reference to electroweak interaction,

$$
\begin{aligned}
& R_{w} \cong \frac{2 G_{w} M_{w f}}{c^{2}} \cong 6.7494 \times 10^{-19} \mathrm{~m} \\
& \text { Schwarzschild radius of } M_{w} \\
& \frac{R_{p}}{R_{w}} \cong\left(\frac{2 G_{s} m_{p}}{c^{2}}\right) \div\left(\frac{2 G_{w} M_{w f}}{c^{2}}\right) \cong \frac{G_{s} m_{p}}{G_{w} M_{w f}} \cong\left(\frac{m_{p}}{m_{e}}\right)
\end{aligned}
$$

With reference to $R_{w} \cong 6.7494 \times 10^{-19} \mathrm{~m}$ and cconsidering $\left(\frac{m_{p}}{m_{e}}\right)$ as a geometric ratio, nuclear radius and atomic radius can be estimated in the following way.

$$
\begin{aligned}
& R_{1} \cong\left(\frac{m_{p}}{m_{e}}\right)\left(\frac{2 G_{w} M_{w f}}{c^{2}}\right) \cong 1.2393 \mathrm{fm} \\
& R_{2} \cong\left(\frac{m_{p}}{m_{e}}\right)^{2}\left(\frac{2 G_{w} M_{w f}}{c^{2}}\right) \cong 2.275 \mathrm{pm}
\end{aligned}
$$

With reference to electromagnetic gravitational constant, Schwarzschild radius of electron can be addressed with,

$$
R_{e} \cong\left(\frac{2 G_{e} m_{e}}{c^{2}}\right) \cong 0.48 \mathrm{~nm}
$$

Based on relations (17) and (18) and identifying $R_{2}$ and $R_{e}$ as characteristic length scales associated with characteristic atomic radius, we noticed that, 


$$
\begin{aligned}
& \sqrt{R_{2} R_{e}} \cong\left(\frac{2 \sqrt{G_{e} G_{s}} m_{p}}{c^{2}}\right) \cong 33.1 \mathrm{pm} \\
& \cong R_{\text {atom }} \cong \text { Scwarzschild radius of atom [43] }
\end{aligned}
$$

\section{Specific unified relations connected with} strong coupling constant

There exists a strong elementary charge $\left(e_{s}\right)$ in such a way that,

$$
\begin{aligned}
& \frac{m_{p}}{m_{e}} \cong\left(\frac{G_{s} m_{p}^{2}}{\hbar c}\right)\left(\frac{G_{e} m_{e}^{2}}{\hbar c}\right) \\
& \left.\cong\left(\frac{e_{s}^{2}}{4 \pi \varepsilon_{0} G_{s} m_{p}^{2}}\right) /\left(\frac{e^{2}}{4 \pi \varepsilon_{0} G_{e} m_{e}^{2}}\right)\right\} \\
& \rightarrow\left\{\begin{array}{l}
\frac{e_{s}^{2}}{e^{2}} \cong\left(\frac{G_{s} m_{p}^{3}}{G_{e} m_{e}^{3}}\right) \cong\left(\frac{G_{s} m_{p}^{2}}{\hbar c}\right)^{2} \cong \frac{1}{\alpha_{s}} \\
\frac{e_{s}}{e} \cong \sqrt{\frac{G_{s} m_{p}^{3}}{G_{e} m_{e}^{3}}} \cong\left(\frac{G_{s} m_{p}^{2}}{\hbar c}\right) \cong \sqrt{\frac{1}{\alpha_{s}}}
\end{array}\right\}
\end{aligned}
$$

where, $\alpha_{s} \cong$ Strong coupling constant

Based on these relations,

$$
\begin{aligned}
& e_{s} \cong 2.9463591 e, \alpha_{s} \cong 0.1151937 \\
& \text { and } \frac{1}{\alpha_{s}} \cong 8.681032
\end{aligned}
$$

\section{Specific unified relations connected with nuclear stability and binding energy}

Nuclear mean stability and binding energy $[44,45]$ can be understood with the following two relations.

Nuclear mean stability can be understood with,

$$
\begin{aligned}
& \left(A_{s}\right)_{\text {mean }} \cong A_{m} \cong 2 Z+k_{1} Z^{2} \\
& \text { where }\left\{\begin{array}{l}
k_{1} \cong 4\left(\frac{G_{s}^{2}}{G_{e} G_{w}}\right) \cong 4\left(\frac{m_{p}}{M_{w f}}\right) \\
\cong 0.0064185
\end{array}\right\}
\end{aligned}
$$

Nuclear binding energy can be understood with,

$$
B_{(A, Z)} \cong\left\{\begin{array}{l}
\left(1-k_{2} \sqrt{Z N}\right) A-A^{1 / 3} \\
-\left(1+\frac{\left(A_{m}-A\right)^{2}}{A_{m}}\right)
\end{array}\right\}\left(B_{0} \cong 10.1 \mathrm{MeV}\right)
$$

$$
\left.\begin{array}{c}
\text { where, } k_{2} \cong 2 \sqrt{\frac{G_{w}}{G_{s}}} \cong 2 \sqrt{\frac{m_{e}}{M_{w f}}} \cong 0.00189 \\
B_{0} \cong\left(\frac{1}{\alpha_{s}}\right) \frac{e^{2}}{4 \pi \varepsilon_{0} R_{0}} \cong \frac{e_{s}^{2}}{4 \pi \varepsilon_{0} R_{0}}
\end{array}\right\}
$$

Note-: The numbers $\left(k_{1}\right.$ and $\left.k_{2}\right)$ can be considered as the characteristic outcomes of the combined effect of strong and electromagnetic coupling constants. With trial-error method, we noticed that, $\left(k_{1}, k_{2}\right) \cong\left(\frac{\left(1-\alpha_{s}\right)^{n}}{2 n-1}\right) \alpha \cong(0.00646,0.0019043)$ where $n \cong 1,2$ and $\alpha_{s} \cong 0.1152$. It needs further study.

\section{Specific unified relations connected with stellar mass limits}

With reference to strong nuclear gravitational constant and astro-physics point of view $[14,16]$, by considering nucleon as a characteristic building block, stellar mass limit $[46,47]$ can be understood with a relation of the form,

$$
\frac{G_{N} M_{X}}{G_{s} m_{n}} \cong \sqrt{\frac{G_{s}}{G_{N}}}
$$

Thus, characteristic stellar mass limit can be estimated with a very simple relation of the form,

$$
M_{X} \cong\left(\frac{G_{s}}{G_{N}}\right)^{\frac{3}{2}}\left(m_{n}\right) \cong 9.37 \text { solar masses }
$$

Another interesting relation is,

$$
\begin{gathered}
\frac{G_{N} M_{X}}{G_{s} \sqrt{m_{n} M_{w f}}} \cong \sqrt{\frac{G_{s}}{G_{N}}} \\
M_{X} \cong\left(\frac{G_{s}}{G_{N}}\right)^{\frac{3}{2}} \sqrt{m_{n} M_{w f}} \cong 234 \text { solar masses }
\end{gathered}
$$


With reference to electromagnetic gravitational constant, mass limits of super massive stellar objects can be understood.

\section{Applications of $G_{e}$ in elementary particle physics and astrophysics}

\section{A) Understanding the recently observed 3.5 keV galactic photon}

Recent galactic X-ray $[48,49]$ studies strongly confirm the existence of a new photon of energy 3.5 $\mathrm{keV}$. So far, its origin is unknown and unclear. In this context, we propose the following alternative mechanism for understanding the origin of $3.5 \mathrm{keV}$ photon.

1) There exists a characteristic charged baby lepton of rest mass,

$$
\left(m_{x l}\right)^{ \pm} \cong \sqrt{\frac{e^{2}}{4 \pi \varepsilon_{0} G_{e}}} \cong 1.75 \mathrm{keV} / c^{2}
$$

2) With pair annihilation mechanism, $\left(m_{x l}\right)$ generates a photon of rest energy $3.5 \mathrm{keV}$

3) With current and future particle accelerators, $\left(m_{x l}\right)^{ \pm} \cong 1.75 \mathrm{keV} / c^{2}$ can be generated.

\section{B) Fitting Muon and Tau rest masses}

Experimentally observed [29] Muon and Tau rest masses can be fitted in the following way.

$$
\left.m_{(\mu, \tau)} c^{2} \cong\left[\gamma^{3}+\left(n^{2} \gamma\right)^{n}\left(\frac{G_{e}}{G_{N}}\right)^{1 / 4}\right]^{\frac{1}{3}} 1.75 \mathrm{keV}\right\}
$$

where,

$\gamma \cong \sqrt{\frac{4 \pi \varepsilon_{0} G_{e} m_{e}^{2}}{e^{2}}} \cong 292.187$ and $n=1$ and 2

For $n=1$, obtained $m_{\mu} c^{2} \cong 106.5 \mathrm{MeV}$

$n=2$, obtained $m_{\tau} c^{2} \cong 1781.5 \mathrm{MeV}$.

At $n=3$, a new heavy charged lepton of rest energy $42.2 \mathrm{GeV}$ can be predicted.

\section{Understanding neutron life time with $\left(G_{e}, G_{w}\right)$}

One of the key objectives of any unified description is to simplify or eliminate the complicated issues of known physics. Neutron life estimation is one of such complicated issue $[29,50,51]$. In this context, in our earlier publications [20,52], we proposed the following relations.

$$
\left.\begin{array}{l}
t_{n} \cong\left(\frac{G_{e}}{G_{w}}\right)\left(\frac{G_{e} m_{n}^{2}}{\left(m_{n}-m_{p}\right) c^{3}}\right) \\
\cong\left(\frac{G_{e}^{2} m_{n}^{2}}{G_{w}\left(m_{n}-m_{p}\right) c^{3}}\right) \cong 874.94 \mathrm{sec}
\end{array}\right\}
$$

Plausible point to be noted is that, relativistic mass of neutron seems to play a crucial role in understanding the increasing neutron life time. It can be understood with,

$$
t_{n} \propto \frac{m_{n}^{2}}{\left[1-\left(v^{2} / c^{2}\right)\right]} \text { and } t_{n} \cong \frac{874.94 \mathrm{sec}}{\left[1-\left(v^{2} / c^{2}\right)\right]}
$$

In this way, bottle method [50] and beam method [51] of neutron life time experiments can be correlated with confined and moving neutrons.

\section{Quark fermions, quark bosons, fluons and bluons}

With reference to our earlier publications on Super Symmetry $[53,54,55,56]$, we propose the following concepts.

\section{A) Quark charge spectrum with respect to Super Symmetry (SUSY)}

1) Strong coupling constant plays a vital role in hadron mass generation.

a) There exists a strong interaction elementary charge $\left(e_{s}\right)$ in such a way that, $e_{s} / e \cong \sqrt{1 / 0.1152} \cong 2.9463 \approx 3$ and $e_{s} \approx 3 e$.

b) With reference to the integral nature of $e$, splitting of $e_{s}$ can be visualized as $\pm e_{s} \rightarrow( \pm e$ and $\pm 2 e)$ $\Rightarrow \pm e= \pm\left(\frac{1}{3} e_{s}\right)$ and $\left.\pm 2 e= \pm\left(\frac{2}{3} e_{s}\right)\right\}$

2) Currently believed basic quarks can be represented by the symbol $\left(m_{q}\right)$ with no second letter in the subscript.

3) Basic quarks can be classified into quark fermions and quark bosons.

4) Mass fraction gained by a quark under super symmetry can be called as 'Quark boson' and can be represented by $\left(m_{q b}\right)$. 
5) Effective quark that lost its fraction of mass to its corresponding boson can be called as 'quark fermion' and can be represented by $\left(m_{q f}\right)$.

6) Quark fermion - boson mass ratio is very close to $\Psi \cong \ln \left(1+\frac{1}{\alpha_{s}}\right) \cong 2.27$ where, $\alpha_{s} \cong 0.1152$.

7) Quark boson mass can be represented by $m_{q b} \cong \frac{m_{q}}{\Psi} \cong \frac{m_{q}}{2.27} \cong 0.4405 m_{q}$.

8) Quark fermion mass can be represented by $\left.\begin{array}{l}m_{q f} \cong m_{q}-m_{q b} \cong m_{q}-\frac{m_{q}}{\Psi} \\ \cong\left(1-\frac{1}{2.27}\right) m_{q} \cong 0.5595 m_{q}\end{array}\right\}$

9) At any level and at any state, if any quark splits into quark fermion and quark boson, due to its heavy mass fraction of $0.5595 m_{q}$, quark fermion carries a strong charge of magnitude $\pm\left(\frac{2}{3} e_{s}\right) \cong \pm 2 e$. Due to its little mass fraction of $0.4405 m_{q}$, quark boson carries a strong charge of magnitude $\pm\left(\frac{1}{3} e_{s}\right) \cong \pm e$.

10) Independent of its level, at any state, strong charge is conserved. Clearly speaking, sum of quark fermion charge and quark boson charge $=\left[ \pm\left(\frac{2}{3} e_{s}\right)\right]+\left[ \pm\left(\frac{1}{3} e_{s}\right)\right] \cong \pm 3 e \cong \pm e_{s}$.

11) At any level, if lower state is assumed to have quark fermions of charge $\mp\left(\frac{1}{3} e_{s}\right)$, then corresponding state quark bosons can have a charge of $\mp\left(\frac{2}{3} e_{s}\right)$.

12) At any level, if upper state is assumed to have quark fermions of charge $\pm\left(\frac{2}{3} e_{s}\right)$, then corresponding state quark bosons can have a charge of $\pm\left(\frac{1}{3} e_{s}\right)$.

13) At any level, electromagnetic charge is conserved. Clearly speaking, charge sum of the two states should always be $\pm\left(\frac{1}{3} e_{s}\right) \cong \pm e$.

14) Up and Down quarks can be considered as first level upper and lower states. At first level, a) Upper state, Up fermion will have a charge of $\pm\left(\frac{2}{3} e_{s}\right)$ and Up boson will have a charge of $\pm\left(\frac{1}{3} e_{s}\right)$.

b) Lower state, Down fermion will have a charge of $\mp\left(\frac{1}{3} e_{s}\right)$ and Down boson will have a charge of $\mp\left(\frac{2}{3} e_{s}\right)$

15) Charm and Strange quarks can be considered as second level upper and lower states. At second level,

a) Upper state, Charm fermion will have a charge of $\pm\left(\frac{2}{3} e_{s}\right)$ and Charm boson will have a charge of $\pm\left(\frac{1}{3} e_{s}\right)$.

b) Lower state, Strange fermion will have a charge of $\mp\left(\frac{1}{3} e_{s}\right)$ and Strange boson will have a charge of $\mp\left(\frac{2}{3} e_{s}\right)$.

16) Top and Bottom quarks can be considered as third level upper and lower states. At third level,

a) Upper state, Top fermion will have a charge of $\pm\left(\frac{2}{3} e_{s}\right)$ and Top boson will have a charge of $\pm\left(\frac{1}{3} e_{s}\right)$.

b) Lower state, Bottom fermion will have a charge of $\mp\left(\frac{1}{3} e_{s}\right)$ and Bottom boson will have a charge of $\mp\left(\frac{2}{3} e_{s}\right)$.

\section{B) Quark mass spectrum with respect to SUSY}

1) Up, Strange and Bottom quarks are in a geometric series with a geometric ratio, $\left.\begin{array}{l}r_{u g} \cong\left(\frac{x(x+1)}{x-1}\right)^{2} \cong(5.8836)^{2} \cong 34.617 \\ \text { where, } x \cong \ln \left(\frac{1}{\alpha_{s}}\right) \cong 2.1611\end{array}\right\}$ 
2) Down, Charm and Top quarks are in another geometric series with a geometric ratio, $\left.\begin{array}{l}r_{d g} \cong\left(\frac{2 x(x+1)}{x-1}\right)^{2} \cong 4 r_{u g} \\ 4 \times(5.8836)^{2} \cong 138.468\end{array}\right\}$

3) Up quark mass can be estimated with $m_{u} \cong\left(\frac{1}{\alpha_{s}}\right) \times m_{e} c^{2} \cong 4.436 \mathrm{MeV}$.

4) Down quark mass can be estimated with $m_{d} \cong x \times m_{u} c^{2} \cong 9.586 \mathrm{MeV}$.

5) There exists a massive fermion of rest energy $\left(M_{h f} c^{2}\right)^{ \pm} \cong 103.4 \mathrm{GeV}$. It can be called as 'baryon mass generator' and needs a formula for its estimation. Roughly, it can be related with the following empirical relation,

$$
\left\{\begin{array}{l}
M_{h f} \cong\left(\frac{e_{s}}{e}\right)^{2} *\left(M_{w f}^{2} m_{u f}\right)^{\frac{1}{3}} \\
\cong 9 *\left(M_{w f}^{2} m_{u f}\right)^{\frac{1}{3}} \cong 103.403 \mathrm{GeV}
\end{array}\right\}
$$

6) Quark fermions that generate observable massive baryons can be called as 'Fluons'. They can be represented by $\left(M_{q f}\right)$.

7) Fluons rest mass can be estimated with a relation of the form, $M_{q f} \cong \frac{1}{2 x}\left[m_{q f} \times M_{h f}^{2}\right]^{\frac{1}{3}}$ where $\frac{1}{2 x} \cong \sin ^{2} \theta_{w} \cong 0.23137$.

8) Obeying the concept of 'fractional quark charge', there exist two kinds of ground state baryons.

9) Type-1 ground state baryons can be addressed with $\left(M_{q f 1} \times M_{q f 2} \times M_{q f 3}\right)^{\frac{1}{3}} \quad$ where $\left(M_{q f 1}, M_{q f 2}\right.$ and $\left.\mathrm{M}_{q f 3}\right)$ represent any three fluons.

10) Type-2 ground state baryons can be addressed with $\left(M_{q f 1}^{2} \times M_{q f 2}\right)^{\frac{1}{3}}$ where $\left(M_{q f 1}\right.$ and $\left.M_{q f 2}\right)$ represent any two fluons.

11) There exist two basic types of excited levels. They can be called as 'fine rotational levels' and 'super fine rotational levels'.

12) Fine rotational levels of ground state baryons can be represented by, $[I=n(n+1)]^{\frac{1}{4}}$ or $[I / 2=n(n+1) / 2]^{\frac{1}{4}} \quad$ where $n=1,2,3$. .

13) Super fine rotational levels of ground state baryons can be represented by

$$
\begin{aligned}
& {[I=n(n+1)]^{\frac{1}{12}} \text { or }[I / 2=n(n+1) / 2]^{\frac{1}{12}} \text { where }} \\
& n=1,2,3, . .
\end{aligned}
$$

14) Fine rotational levels seem to be associated with nucleons at low energy scales and super fine rotational levels seem to be associated with fluons and bluons. It needs further study.

15) There exists a massive boson of rest energy $\left(M_{h b} c^{2}\right)^{ \pm} \cong \frac{103.403 \mathrm{GeV}}{2.27} \simeq 45.552 \mathrm{GeV}$. It can be called as 'meson mass generator'.

16) Quark bosons that generate observable mesons can be called as 'Bluons'. They can be represented by $\left(M_{q b}\right)$.

17) Bluons rest mass can be estimated with a relation of the form, $M_{q b} \cong \frac{1}{2 x}\left[m_{q b} \times M_{h b}^{2}\right]^{\frac{1}{3}}$.

18) Obeying the concept of 'fractional quark charge', mesons can be understood in three different ways and needs further in depth study.

19) Type-1 ground state mesons can be addressed with $\quad\left(M_{q b 1}+M_{q b 2}\right)$ or $\sqrt{M_{q b 1} M_{q b 2}} \quad$ where $\left(M_{q b 1}\right.$ and $\left.M_{q b 2}\right)$ represent any two bluons.

20) Type-2 ground state mesons can be addressed with $\quad\left(M_{q f 1} \times M_{q f 2} \times M_{q f 3}\right)^{\frac{1}{3}} / 2.27 \quad$ or $\left(M_{q f 1}^{2} \times M_{q f 2}\right)^{\frac{1}{3}} / 2.27$. It needs a review.

21) Super fine rotational levels of ground state mesons can be represented by $[I=n(n+1)]^{\frac{1}{12}}$ or $[I / 2=n(n+1) / 2]^{\frac{1}{12}}$ where $n=1,2,3$, ..

\section{C) Quark mass and charge spectrum}

See the following tables pertaining to quark masses and charges.

\begin{tabular}{|c|c|c|}
\hline \multicolumn{3}{|c|}{ Table-1: Basic quark masses and strong charge } \\
\hline Basic quark & $\begin{array}{c}\left(m_{q}\right) \text { Mass } \\
(\mathrm{MeV})\end{array}$ & $\begin{array}{c}\text { Strong } \\
\text { Charge }\end{array}$ \\
\hline Up & 4.44 & $\pm e_{s}= \pm 3 e$ \\
\hline Down & 9.59 & $\pm e_{s}= \pm 3 e$ \\
\hline Strange & 153.55 & $\pm e_{s}= \pm 3 e$ \\
\hline Charm & 1327.36 & $\pm e_{s}= \pm 3 e$ \\
\hline Bottom & 5315.50 & $\pm e_{s}= \pm 3 e$ \\
\hline Top & 183796.1 & $\pm e_{s}= \pm 3 e$ \\
\hline
\end{tabular}




\begin{tabular}{|c|c|c|}
\hline \multicolumn{2}{|c|}{ Table-2: Quark fermion masses and strong charges } \\
\hline $\begin{array}{c}\text { Quark } \\
\text { fermion }\end{array}$ & $\begin{array}{c}\left(m_{q f}\right) \text { Mass } \\
(\mathrm{MeV})\end{array}$ & $\begin{array}{c}\text { Strong } \\
\text { Charge }\end{array}$ \\
\hline Up & 2.48 & $\left( \pm \frac{2 e_{s}}{3}\right)$ \\
\hline Down & 5.37 & $\left( \pm \frac{2 e_{s}}{3}\right)$ \\
\hline Charm & 742.66 & $\left(\mp \frac{e_{s}}{3}\right)$ \\
\hline Strange & 85.91 & $\left(\mp \frac{e_{s}}{3}\right)$ \\
\hline Bottom & 2974.02 & $\left( \pm \frac{2 e_{s}}{3}\right)$ \\
\hline Top & 102833.92 & \\
\hline
\end{tabular}

\begin{tabular}{|c|c|c|}
\hline Charm & 4615.82 & $\left( \pm \frac{2 e_{s}}{3}\right)$ \\
\hline Strange & 2249.10 & $\left(\mp \frac{e_{s}}{3}\right)$ \\
\hline Top & 23880.18 & $\left( \pm \frac{2 e_{s}}{3}\right)$ \\
\hline Bottom & 7330.05 & $\left(\mp \frac{e_{s}}{3}\right)$ \\
\hline
\end{tabular}

\begin{tabular}{|c|c|c|}
\hline \multicolumn{2}{|c|}{ Table-3: Quark boson masses and strong charges } \\
\hline Quark boson & $\begin{array}{c}\left(m_{q b}\right) \text { Mass } \\
(\mathrm{MeV})\end{array}$ & $\begin{array}{c}\text { Strong } \\
\text { Charge }\end{array}$ \\
\hline Up & 1.96 & $\left( \pm \frac{e_{s}}{3}\right)$ \\
\hline Down & 4.22 & $\left( \pm \frac{2 e_{s}}{3}\right)$ \\
\hline Charm & 584.70 & $\left(\mp \frac{2 e_{s}}{3}\right)$ \\
\hline Strange & 67.64 & $\left( \pm \frac{e_{s}}{3}\right)$ \\
\hline Top & 80962.18 & $\left(\mp \frac{2 e_{s}}{3}\right)$ \\
\hline Bottom & 2341.48 & $(\mp 8$ \\
\hline
\end{tabular}

\begin{tabular}{|c|c|c|}
\hline Table-5: Bluon masses and strong charges \\
\hline Bluons & $\begin{array}{c}\left(M_{q b}\right) \text { Mass } \\
(\mathrm{MeV})\end{array}$ & $\begin{array}{c}\text { Strong } \\
\text { Charge }\end{array}$ \\
\hline Up & 369.04 & $\left( \pm \frac{e_{s}}{3}\right)$ \\
\hline Down & 477.04 & $\left(\mp \frac{2 e_{s}}{3}\right)$ \\
\hline Charm & 2467.58 & $\left( \pm \frac{e_{s}}{3}\right)$ \\
\hline Strange & 1202.35 & $\left(\mp \frac{2 e_{s}}{3}\right)$ \\
\hline Top & 12766.16 & $\left( \pm \frac{e_{s}}{3}\right)$ \\
\hline Bottom & 3918.59 & $\left(\mp \frac{2 e_{s}}{3}\right)$ \\
\hline
\end{tabular}

\section{Discussion on baryon masses}

From Table-4, the following baryon ground state masses can be estimated. See Table-6.

\begin{tabular}{|l|c|c|}
\hline \multicolumn{3}{|c|}{ Table-6: Ground state mass of baryons } \\
\hline $\begin{array}{c}\text { Combination of } \\
\text { fluons }\end{array}$ & $\begin{array}{c}\text { Ground state } \\
\text { mass of baryon } \\
(\mathrm{MeV})\end{array}$ & $\begin{array}{c}\text { Electromagnetic } \\
\text { Charge of } \\
\text { baryon }\end{array}$ \\
\hline$\left(M_{u f}^{2} M_{d f}\right)^{\frac{1}{3}}$ & 751.99 & $(+e)$ \\
\hline$\left(M_{d f}^{2} M_{u f}\right)^{\frac{1}{3}}$ & 819.63 & $(0)$ \\
\hline$\left(M_{u f}^{2} M_{s f}\right)^{\frac{1}{3}}$ & 1023.38 & $(+e)$ \\
\hline$\left(M_{u f} M_{d f} M_{s f}\right)^{\frac{1}{3}}$ & 1114.80 & $(0)$ \\
\hline$\left(M_{d f}^{2} M_{s f}\right)^{\frac{1}{3}}$ & 1214.38 & $(-e)$ \\
\hline
\end{tabular}




\begin{tabular}{|c|c|c|c|c|c|}
\hline$\left(M_{s f}^{2} M_{u f}\right)^{\frac{1}{3}}$ & 1517.13 & $(0)$ & $\left(M_{t f}^{2} M_{c f}\right)^{\frac{1}{3}}$ & 13807.28 & $(+2 e)$ \\
\hline$\left(M_{s f}^{2} M_{d f}\right)^{\frac{1}{3}}$ & 1652.66 & $(-e)$ & $\left(M_{t f}^{2} M_{b f}\right)^{\frac{1}{3}}$ & 16108.71 & $(+e)$ \\
\hline$\left(M_{u f}^{2} M_{c f}\right)^{\frac{1}{3}}$ & 1300.52 & $(+2 e)$ & $\left(M_{u f} M_{d f} M_{t f}\right)^{\frac{1}{3}}$ & 2450.23 & $(+e)$ \\
\hline$\left(M_{d f}^{2} M_{c f}\right)^{\frac{1}{3}}$ & 1543.25 & (0) & $\left(M_{u f} M_{s f} M_{t f}\right)^{\frac{1}{3}}$ & 3334.51 & $(+e)$ \\
\hline$\left(M_{s f}^{2} M_{c f}\right)^{\frac{1}{3}}$ & 2858.17 & $(0)$ & $\left(M_{u f} M_{c f} M_{t f}\right)^{\frac{1}{3}}$ & 4237.52 & $(+2 e)$ \\
\hline$\left(M_{c f}^{2} M_{u f}\right)^{\frac{1}{3}}$ & 2450.1 & $(+2 e)$ & $\left(M_{u f} M_{b f} M_{t f}\right)^{\frac{1}{3}}$ & 4943.85 & $(+e)$ \\
\hline$\left(M_{c f}^{2} M_{d f}\right)^{\frac{1}{3}}$ & 2668.96 & $(+e)$ & $\left(M_{d f} M_{s f} M_{t f}\right)^{\frac{1}{3}}$ & 3632.39 & (0) \\
\hline$\left(M_{c f}^{2} M_{s f}\right)^{\frac{1}{3}}$ & 3632.19 & $(+e)$ & $\left(M_{d f} M_{c f} M_{t f}\right)^{\frac{1}{3}}$ & 4616.07 & $(+e)$ \\
\hline$\left(M_{u f} M_{d f} M_{c f}\right)^{\frac{1}{3}}$ & 1416.70 & $(+e)$ & $\left(M_{d f} M_{b f} M_{t f}\right)^{\frac{1}{3}}$ & 5385.49 & (0) \\
\hline$\left(M_{u f} M_{s f} M_{c f}\right)^{\frac{1}{3}}$ & 1927.98 & $(+e)$ & $\left(M_{s f} M_{b f} M_{t f}\right)^{\frac{1}{3}}$ & 7329.11 & $(+e)$ \\
\hline$\left(M_{d f} M_{s f} M_{c f}\right)^{\frac{1}{3}}$ & 2100.21 & $(0)$ & $\left(M_{s f} M_{c f} M_{t f}\right)^{\frac{1}{3}}$ & 6282.00 & $(+e)$ \\
\hline$\left(M_{u f}^{2} M_{b f}\right)^{\frac{1}{3}}$ & 1517.29 & $(+e)$ & $\left(M_{b f}^{2} M_{t f}\right)^{\frac{1}{3}}$ & 10866.35 & $(0)$ \\
\hline$\left(M_{d f}^{2} M_{b f}\right)^{\frac{1}{3}}$ & 1800.48 & $(-e)$ & $\left(M_{b f} M_{c f} M_{t f}\right)^{\frac{1}{3}}$ & 9313.89 & $(+e)$ \\
\hline$\left(M_{s f}^{2} M_{b f}\right)^{\frac{1}{3}}$ & 3334.58 & $(-e)$ & $\left(M_{c f}^{2} M_{t f}\right)^{\frac{1}{3}}$ & 7983.23 & $(+2 e)$ \\
\hline
\end{tabular}

We assure the readers that, with these ground state baryon masses and considering their super fine rotational levels, most of the observed baryons can be fitted and many new states can also be predicted for future experimental verification/observation. There may be some minor differences in the current classification scheme and proposed scheme of baryon super symmetry.

\section{Discussion on meson masses}

From Table-5, the following ground state meson masses can be estimated. See Table-7.

\begin{tabular}{|l|c|c|}
\hline \multicolumn{3}{|c|}{ Table-7: Ground state mass of mesons } \\
\hline $\begin{array}{c}\text { Combination of } \\
\text { bluons }\end{array}$ & $\begin{array}{c}\text { Ground state } \\
\text { mass of meson } \\
(\mathrm{MeV})\end{array}$ & $\begin{array}{c}\text { Electromagnetic } \\
\text { Charge of } \\
\text { meson }\end{array}$ \\
\hline$M_{u b}+\bar{M}_{u b}$ & 738.08 & $(0)$ \\
\hline$M_{d b}+\bar{M}_{u b}$ & 846.08 & $(-e)$ \\
\hline$M_{d b}+\bar{M}_{d b}$ & 954.08 & $(0)$ \\
\hline$M_{s b}+\bar{M}_{u b}$ & 1571.39 & $(-e)$ \\
\hline$M_{s b}+\bar{M}_{d b}$ & 1679.39 & $(0)$ \\
\hline$M_{s b}+\bar{M}_{s b}$ & 2404.7 & $(0)$ \\
\hline
\end{tabular}




\begin{tabular}{|l|c|c|}
\hline$M_{c b}+\overline{M_{u b}}$ & 2836.62 & $(0)$ \\
\hline$M_{c b}+\bar{M}_{a b}$ & 2944.62 & $(+e)$ \\
\hline$M_{c b}+\bar{M}_{s b}$ & 3669.93 & $(+e)$ \\
\hline$M_{b b}+\bar{M}_{u b}$ & 4287.63 & $(+e)$ \\
\hline$M_{b b}+\bar{M}_{d b}$ & 4395.63 & $(0)$ \\
\hline$M_{b b}+\overline{M_{s b}}$ & 5120.94 & $(0)$ \\
\hline$M_{b b}+\overline{M_{c b}}$ & 6386.17 & $(-e)$ \\
\hline$M_{b b}+\overline{M_{b b}}$ & 7837.18 & $(0)$ \\
\hline$M_{t b}+\overline{M_{u b}}$ & 13135.2 & $(0)$ \\
\hline$M_{t b}+\overline{M_{d b}}$ & 13243.2 & $(+e)$ \\
\hline$M_{t b}+\overline{M_{s b}}$ & 13968.51 & $(+e)$ \\
\hline$M_{t b}+\overline{M_{b b}}$ & 16684.75 & $(+e)$ \\
\hline$M_{t b}+\overline{M_{t b}}$ & 25532.32 & $(0)$ \\
\hline
\end{tabular}

Apart from these ground states, by considering $\sqrt{M_{q b 1} M_{q b 2}}$, other ground states can also predicted. See the following Table- 8 for the charged ground states.

\begin{tabular}{|l|c|c|}
\hline \multicolumn{4}{|c|}{ Table-8: Ground state mass of mesons } \\
\hline $\begin{array}{c}\text { Combination of } \\
\text { bluons }\end{array}$ & $\begin{array}{c}\text { Ground state } \\
\text { mass of meson } \\
\text { (MeV) }\end{array}$ & $\begin{array}{c}\text { Electromagnetic } \\
\text { Charge of } \\
\text { meson }\end{array}$ \\
\hline$\sqrt{M_{a b} \times \overline{M_{a b}}}$ & 419.58 & $(-e)$ \\
\hline$\sqrt{M_{s b} \times \overline{M_{u b}}}$ & 666.12 & $(-e)$ \\
\hline$\sqrt{M_{c b} \times \overline{M_{a b}}}$ & 1084.96 & $(+e)$ \\
\hline$\sqrt{M_{c b} \times \overline{M_{s b}}}$ & 1722.47 & $(+e)$ \\
\hline$\sqrt{M_{b b} \times \overline{M_{u b}}}$ & 1202.55 & $(+e)$ \\
\hline$\sqrt{M_{b b} \times \overline{M_{c b}}}$ & 3109.57 & $(-e)$ \\
\hline$\sqrt{M_{t b} \times \overline{M_{a b}}}$ & 2467.78 & $(+e)$ \\
\hline$\sqrt{M_{t b} \times \overline{M_{s b}}}$ & 3917.83 & $(+e)$ \\
\hline$\sqrt{M_{t b} \times \overline{M_{b b}}}$ & 7072.86 & $(+e)$ \\
\hline
\end{tabular}

\section{General discussion}

We appeal that,

(1) Success of any unified model depends on its ability to involve gravity in microscopic models.

(2) Full-fledged implementation of gravity in microscopic physics must be able to:

a) Estimate the ground state elementary particle rest masses of the three atomic interactions.

b) Estimate the coupling constants of the three atomic interactions.

c) Estimate the range of all interactions.

d) Estimate the Newtonian gravitational constant.

(3) As the root/path is unclear and unknown, to make it success or to have a full-fledged implementation, one may be forced to consider a new path that may be out-of-scope of the currently believed string theory models [57] or super symmetric models [58-61].

(4) In our approach,

a) We assign a different gravitational constant for each basic interaction.

b) Considering $585 \mathrm{GeV}$ fermion as the characteristic building block of all elementary particles, an attempt is made to fit proton and electron masses.

c) During this journey, without considering arbitrary numbers or coefficients, we come across many strange and interesting relations for estimating other atomic and nuclear coupling constants.

d) Based on relations (5) and (6), magnitudes of $\left(G_{w}, \alpha_{s}\right)$ can be estimated in a verifiable approach.

e) Based on super symmetry, in sections (13 to 15), we have presented a brief report on baryons and mesons. It needs further study. In this context, we are working and trying to seek field experts' opinion and guidelines.

(5) Based on the proposed concept of SUSY, corresponding to the proposed electroweak fermion of rest energy $\left(M_{w j} c^{2}\right)^{ \pm} \cong 584.725 \mathrm{GeV}$, there exists a possibility of finding a characteristic electroweak boson of rest energy, $\left(M_{w b} c^{2}\right)^{ \pm} \cong \frac{\left(M_{w f} c^{2}\right)^{ \pm}}{\Psi} \cong 257.59 \mathrm{GeV}$

(6) In our earlier publications [53,54], we expressed our view that,

a) Charged electroweak boson $\left(M_{W}\right)^{ \pm}$is a super symmetric boson of "integral" charge top quark. In this paper, we have reviewed our earlier paper with respect to 'strong' charge and fractional charge quark bosons. By assuming the combination of Top quark boson 
and anti Strange boson or anti Down boson, there is a possibility of observing $\left(M_{W}\right)^{ \pm}$. If so, there is also a possibility of observing neutral boson of rest energy close to $\left(M_{W}\right)^{ \pm}$ boson. Hence, it needs further study with respect to the fractional charge quark boson of rest energy of $80.9 \mathrm{GeV}$.

b) Neutral electroweak boson constitutes two charged bosons of rest energy $(45.5 \mathrm{GeV})^{ \pm}$. It can be expressed as,

$$
\begin{aligned}
& M_{Z} \cong\left(\frac{M_{h f}}{\Psi}\right)^{ \pm}+\left(\frac{M_{h f}}{\Psi}\right)^{\mp} \\
& \cong\left(M_{h b}\right)^{ \pm}+\left(M_{h b}\right)^{\mp} \cong \frac{2 M_{h f}}{\Psi} \cong 91.104 \mathrm{GeV} / c^{2}
\end{aligned}
$$

c) Neutral Higg's boson constitutes $[23,62]$ a charged electroweak boson $\left(M_{W}\right)^{ \pm}$and a charged $\left(M_{h b}\right)^{\mp}$. It can be expressed as,

$$
\begin{aligned}
\left(M_{H}\right)^{0} & \cong\left(M_{W}\right)^{ \pm}+\left(M_{h b}\right)^{\mp} \\
& \cong(80.379+45.552) \mathrm{GeV} / c^{2} \\
& \cong 125.93 \mathrm{GeV} / c^{2}
\end{aligned}
$$

d) With reference to the data presented in Table-3, neutral pion seems to constitute Strange bluon and anti Strange bluon. It's rest energy seems to be $2 * 67.64=135.3 \mathrm{MeV}$.

\begin{tabular}{|c|c|c|c|}
\hline \multicolumn{4}{|c|}{$\begin{array}{l}\text { Table-9: Super fine rotational levels of Charm bluon } \\
\text { and anti Up bluon.(Neutral charge) }\end{array}$} \\
\hline$n$ & $n(n+1)$ & $\begin{array}{c}{[n(n+1)]^{\frac{1}{12}}} \\
(\mathrm{MeV})\end{array}$ & $\begin{array}{c}{[n(n+1) / 2]^{\frac{1}{12}}} \\
(\mathrm{MeV})\end{array}$ \\
\hline 1 & 2 & 3005.3 & 2836.6 \\
\hline 2 & 6 & 3293.4 & 3108.6 \\
\hline 3 & 12 & 3489.3 & 3293.4 \\
\hline 4 & 20 & 3641.0 & 3436.6 \\
\hline 5 & 30 & 3766.1 & 3554.7 \\
\hline 6 & 42 & 3873.2 & 3655.8 \\
\hline 7 & 56 & 3967.2 & 3744.5 \\
\hline 8 & 72 & 4051.2 & 3823.8 \\
\hline 9 & 90 & 4127.2 & 3895.6 \\
\hline 10 & 110 & 4196.8 & 3961.2 \\
\hline 11 & 132 & 4261.0 & 4021.9 \\
\hline 12 & 156 & 4320.8 & 4078.3 \\
\hline 13 & 182 & 4376.6 & 4131.0 \\
\hline 14 & 210 & 4429.1 & 4180.6 \\
\hline 15 & 240 & 4478.7 & 4227.3 \\
\hline
\end{tabular}

e) Currently believed Charm and anti Charm mesons can be understood with super fine rotational levels of Charm bluon and anti Up bluon. See Table -9.

\begin{tabular}{|l|l|l|l|}
16 & 272 & 4525.7 & 4271.7 \\
\hline 17 & 306 & 4570.3 & 4313.8 \\
\hline 18 & 342 & 4612.9 & 4354.0 \\
\hline 19 & 380 & 4653.5 & 4392.4 \\
\hline 20 & 420 & 4692.5 & 4429.1 \\
\hline
\end{tabular}

f) Similarly currently believed charged Strange and Charm mesons can be understood with super fine rotational levels of Charm bluon and anti Strange bluon. See Table-10.

\begin{tabular}{|c|c|c|c|}
\hline \multicolumn{3}{|c|}{$\begin{array}{c}\text { Table-10: Super fine rotational levels of Charm } \\
\text { bluon and anti Strange bluon. }( \pm e)\end{array}$} \\
\hline$n$ & $n(n+1)$ & $\begin{array}{c}{[n(n+1)]^{\frac{1}{12}}} \\
(\mathrm{MeV})\end{array}$ & $\begin{array}{c}{[n(n+1) / 2]^{\frac{1}{12}}} \\
(\mathrm{MeV})\end{array}$ \\
\hline 1 & 2 & 1824.9 & $\mathbf{1 7 2 2 . 5}$ \\
\hline 2 & 6 & 1999.9 & 1887.6 \\
\hline 3 & 12 & 2118.8 & 1999.9 \\
\hline 4 & 20 & 2210.9 & 2086.8 \\
\hline 5 & 30 & 2286.9 & 2158.5 \\
\hline 6 & 42 & 2351.9 & 2219.9 \\
\hline 7 & 56 & 2409.0 & 2273.8 \\
\hline 8 & 72 & 2460.0 & 2321.9 \\
\hline 9 & 90 & 2506.1 & 2365.5 \\
\hline 10 & 110 & 2548.4 & 2405.4 \\
\hline 11 & 132 & 2587.4 & 2442.2 \\
\hline 12 & 156 & 2623.7 & 2476.4 \\
\hline 13 & 182 & 2657.6 & 2508.4 \\
\hline 14 & 210 & 2689.5 & 2538.5 \\
\hline 15 & 240 & 2719.6 & 2566.9 \\
\hline 16 & 272 & 2748.1 & 2593.9 \\
\hline 17 & 306 & 2775.2 & 2619.4 \\
\hline 18 & 342 & 2801.0 & 2643.8 \\
\hline 19 & 380 & 2825.7 & 2667.2 \\
\hline 20 & 420 & 2849.4 & 2689.5 \\
\hline & & & \\
\hline
\end{tabular}

g) Considering 'nuclear space' as a sea of -

i. Six fractional charge quark bosons of rest energy $2 \mathrm{MeV}$ to $81 \mathrm{GeV}$,

ii. Six fractional charge quark fermions of rest energy $2.5 \mathrm{MeV}$ to $103 \mathrm{GeV}$,

iii. Six fractional charge fluons of rest energy 692 $\mathrm{MeV}$ to $24 \mathrm{GeV}$ and

iv. Six fractional charge bluons of rest energy 369 $\mathrm{MeV}$ to $13 \mathrm{GeV}$

Many interesting things can be expected.

\section{Conclusion}

With further study, research and confirming the existence of the proposed $\left(M_{w f} c^{2}\right)^{ \pm} \cong 584.725 \mathrm{GeV}$, 
or confirming the existence of its corresponding SUSY boson, $\left(M_{w b} c^{2}\right)^{ \pm} \cong \frac{\left(M_{w f} c^{2}\right)^{ \pm}}{\Psi} \cong 257.59 \mathrm{GeV}$, actual essence of final unification can be understood. Proceeding further, by correlating the currently believed baryons and mesons with the proposed scheme of hadronic super symmetry, further research can be carried out.

\section{Acknowledgements}

Author Seshavatharam is indebted to professors shri M. Nagaphani Sarma, Chairman, shri K.V. Krishna Murthy, founder Chairman, Institute of Scientific Research in Vedas (I-SERVE), Hyderabad, India and Shri K.V.R.S. Murthy, former scientist IICT (CSIR), Govt. of India, Director, Research and Development, I-SERVE, for their valuable guidance and great support in developing this subject.

\section{References}

[1] Frank Wilczek. QCD made simple (PDF). Physics Today. 53 (8): 22-28 (2000)

[2] M. Bojowald. Quantum cosmology: a review. Rep. Prog. Phys. 78 (2015) 023901

[3] Hawking, S.W. Particle Creation by Black Holes. Communications in Mathematical Physics, 43, 199-220(1975)

[4] K. Tennakone, Electron, muon, proton, and strong gravity. Phys. Rev. D, 10, 1722 (1974)

[5] C. Sivaram and K. Sinha, Strong gravity, black holes, and hadrons. Physical Review D., 16(6), 1975-1978 (1977)

[6] De Sabbata V and M. Gasperini. Strong gravity and weak interactions. Gen. Relat. Gravit. 10, 9, 731-741, (1979)

[7] Salam A, Sivaram C. Strong Gravity Approach to QCD and Confinement. Mod. Phys. Lett., v. A8(4), 321-326 (1993)

[8] Roberto Onofrio. On weak interactions as shortdistance manifestations of gravity. Modern Physics Letters A 28, 1350022 (2013)

[9] Seshavatharam UVS and Lakshminarayana S. A Practical Model of Godel-Planck- Hubble-Birch Universe. Athens Journal of Sciences- Vol.6, Issue 3, 211-230 (2019)

[10] Seshavatharam UVS, Lakshminarayana S. To confirm the existence of atomic gravitational constant. Hadronic Journal.34(4):379 (2011)

[11] Seshavatharam UVS, Lakshminarayana S. Molar electron mass and the basics of TOE. Journal of Nuclear and Particle Physics. 2(6):132 -141 (2012)
[12] Seshavatharam UVS et al. Understanding the constructional features of materialistic atoms in the light of strong nuclear gravitational coupling. Materials Today: 3/10PB, Proceedings 3, 39763981 (2016)

[13] Seshavatharam UVS and Lakshminarayana S. Towards a workable model of final unification. International Journal of Mathematics and Physics 7(1), 117-130 (2016)

[14] Seshavatharam UVS and Lakshminarayana S. Understanding the basics of final unification with three gravitational constants associated with nuclear, electromagnetic and gravitational interactions. Journal of Nuclear Physics, Material Sciences, Radiation and Applications 4(1),1-19 (2017)

[15] Seshavatharam UVS and Lakshminarayana S. On the role of 'reciprocal' of the strong coupling constant in nuclear structure. Journal of Nuclear Sciences, 4( 2), 31-44 (2017).

[16] Seshavatharam UVS and Lakshminarayana S. Applications of gravitational model of possible final unification in both large and small scale physics. Prespacetime journal, Vol 7, issue2, 405-421 (2016)

[17] Seshavatharam UVS and Lakshminarayana S. A virtual model of microscopic quantum gravity, Prespacetime Journal, 9(1), 58-82 (2018)

[18] Seshavatharam UVS and Lakshminarayana S. On the role of four gravitational constants in nuclear structure. Mapana Journal of Sciences, 18(1), 2145 (2019)

[19] Seshavatharam UVS and Lakshminarayana S. On the Role of Large Nuclear Gravity in Understanding Strong Coupling Constant, Nuclear Stability Range, Binding Energy of Isotopes and Magic proton numbers - A Critical Review. J. Nucl. Phys. Mat. Sci. Rad. A. 6(2), 142-160 (2019)

[20] Seshavatharam UVS and Lakshminarayana S. On the Role of Squared Neutron Number in Reducing Nuclear Binding Energy in the Light of Electromagnetic, Weak and Nuclear Gravitational Constants - A Review. Asian Journal of Research and Reviews in Physics, 2(3): 1-22, (2019)

[21]F. Englert and R. Brout. Broken Symmetry and the Mass of Gauge Vector Mesons. Physical Review Letters, vol. 13, Issue 9, pp. 321-323 (1964)

[22] Higgs P. Broken Symmetries and the Masses of Gauge Bosons. Physical Review Letters. 13 (16): 508-509 (1964)

[23] The ATLAS Collaboration. Observation of a new particle in the search for the Standard Model Higgs boson with the ATLAS detector at the LHC. Phys.Lett. B716, 1-29 (2012) 
[24]Ralph A. Alpher, George Gamow and Robert Herman.Thermal cosmic radiation and the formation of protogalaxies. PNAS, 58 (6) 21792186. (1967)

[25]G. Gamow. The Evolution of the Universe. Nature 162, 680-682 (1948)

[26] Berezhiani L, Khoury J and Wang J. Universe without Dark Energy: Cosmic Acceleration from Dark Matter-baryon Interactions. Physical Review 95(12-15): 123530 (2017)

[27] Max Planck. Ueber das Gesetz der Energieverteilung im Normalspectrum (PDF). Ann. Phys., 309 (3): 553-63 (1901)

[28] Niels Bohr. On the Constitution of Atoms and Molecules, Part I (PDF). Philosophical Magazine. 26 (151): 1-24 Niels Bohr (1913)

[29] M. Tanabashi et al. (Particle Data Group), Phys. Rev. D 98, 030001 (2018)

[30]Fermi E. Tentativo di una teoria dei raggi $\beta$. La Ricerca Scientifica (in Italian). 2 (12), (1933).

[31] P. J. Mohr, D. B. Newell, and B. N. Taylor, CODATA recommended values of the fundamental constants: Rev. Mod. Phys. 88, 035009 (2014)

[32] B. Canuel et al. Exploring gravity with the MIGA large scale atom interferometer. Science reports, $8: 14064$ (2018)

[33]Christos Merkatas et al. Shades of dark uncertainty and consensus value for the Newtonian constant of gravitation. arXiv:1905.09551v1 (2019)

[34] Li, Qing et al. Measurements of the gravitational constant using two independent methods. Nature 560, 582-588 (2018)

[35]G. Rosi, F. Sorrentino, L. Cacciapuoti, M. Prevedelli, and G. M. Tino. Precision measurement of the Newtonian gravitational constant using cold atoms, Nature 510 (7506), 518-521 (2014)

[36] S. Schlamminger and R.D. Newman. Recent measurements of the gravitational constant as a function of time. Phys. Rev. D 91, 121101 (2015)

[37]C. Rothleitner and S. Schlamminger. Measurements of the Newtonian constant of gravitation, G. Rev. Sci. Instrum. 88, 111101 (2017)

[38] G. Rosi, Challenging the big $G$ measurement with atoms and light. J. Phys. B: At., Mol. Opt. Phys. 49(20), 202002 (2016)

[39] Seshavatharam UVS and Lakshminarayana S. On the role of Newtonian gravitational constant in estimating Proton-Electron mass ratio and baryon mass spectrum. International Journal of Innovative Studies in Sciences Engineering Technology. Vol 5, Issue 10, 18-21 (2019)

[40]Rutherford E. The scattering of $\alpha$ and $\beta$ particles by matter and the structure of the atom. The London, Edinburgh, and Dublin Philosophical Magazine and Journal of Science. Series 6. 21 (125): (1911)

[41] Hofstadter R. et al. High-Energy Electron Scattering and Nuclear Structure Determinations. Phys. Rev. 92, 978 (1953)

[42] T. Bayram, S. Akkoyun, S. O. Kara and A. Sinan, New Parameters for Nuclear Charge Radius Formulas, Acta Physica Polonica B. 44( 8), 17911799 (2013)

[43] https://en.wikipedia.org/wiki/Atomic_radii_of_th e_elements_(data_page)

[44] Seshavatharam UVS and Lakshminarayana S. Implications and Applications of Fermi Scale Quantum Gravity. Preprints 2019, 2019110134.

[45] Seshavatharam UVS and Lakshminarayana S. Hypothetical Role of Large Nuclear Gravity in Understanding the Significance and Applications of the Strong Coupling Constant in the Light of Up and Down Quark Clusters. Preprints 2019, 2019110398.

[46] Astrophysics and Space Science Library. 412, Very Massive Stars in the Local Universe. Editor Jorick S. Vink. Springer (2014)

[47] https://openstax.org/books/astronomy/pages/182-measuring-stellar-masses

[48] Anirban Biswas et al. Explaining the $3.5 \mathrm{keV}$ Xray Line in a $L \mu-L \tau$ Extension of the Inert Doublet Model. JCAP 02, 002 (2018)

[49] Nico Cappelluti et al. Searching for the $3.5 \mathrm{keV}$ Line in the Deep Fields with Chandra: The $10 \mathrm{Ms}$ Observations. The Astrophysical Journal, 854:179 (12pp), (2018)

[50]RW Pattie Jr et al. Measurement of the neutron lifetime using a magneto-gravitational trap and in situ detection. Science 11, Vol. 360, Issue 6389, pp. 627-632 (2018)

[51]F. E. Wietfeldt et al. A path to a $0.1 \mathrm{~s}$ neutron lifetime measurement using the beam method. Physics Procedia 51, $54-58$ ( 2014 )

[52] Seshavatharam UVS and Lakshminarayana S. Neutron life time enigma in the light of Electromagnetic and Weak gravitational constants. Nucleus-2019, Dubna, Russia, Book of abstracts, Page 242 (2019)

[53] Seshavatharam UVS and Lakshminarayana S. Super Symmetry in Strong and Weak interactions. Int. J. Mod. Phys. E, Vol.19, No.2, p.263-280 (2010).

[54] Seshavatharam UVS and Lakshminarayana S. SUSY and strong nuclear gravity in (120-160) $\mathrm{GeV}$ mass range. Hadronic journal, Vol-34, No 3, 277 (2011)

[55] Seshavatharam UVS and Lakshminarayana S. Integral charge SUSY in Strong nuclear gravity. 
Proceedings of the DAE Symp. on Nucl. Phys. 56, 842 (2011).

[56] Seshavatharam UVS and Lakshminarayana S. Molar Electron Mass and the Basics of TOE, Journal of Nuclear and Particle Physics, Vol. 2 No. 6, 132 (2012).

[57] Sunil Mukhi. String theory: a perspective over the last 25 years. Class. Quant. Grav. 28 153001(2011)

[58] The Supersymmetric World - The Beginnings of the Theory, World Scientific, Singapore (2000), edited by G. Kane and M. Shifman.

[59]D. Joseph Lykken. Introduction to Supersymmetry. FERMILAB-PUB-96/445-T

[60] Weinberg, Steven, The Quantum Theory of Fields: Supersymmetry, Cambridge University Press, Cambridge, Vol. 3 (1999)

[61] Joseph Polchinski. String theory to the rescue. arXiv:1512.02477v5 [hep-th] (2015)

[62] Patrick Draper et al. Implications of a $125 \mathrm{GeV}$ Higgs for the MSSM and Low-Scale SUSY Breaking. Phys. Rev. D 85, 095007 (2012) 